\title{
Negativlisten und Positivlisten in der Pharmakotherapie älterer Patienten
}

Die Verordnung von Arzneimitteln ist die meist praktizierte therapeutische Maßnahme. In den Industrienationen sind die älteren Menschen (>65 Jahre) die am stärksten wachsende Bevölkerungsgruppe. Beim älteren Patienten bestehen dabei häufig mehrere überlappende, oft chronische Krankheitsprozesse, die Indikationen zu lebensbegleitender Arzneimitteltherapie darstellen können (z.B. Herzinsuffizienz, Hypertonie und Diabetes mellitus, aber auch psychiatrische Krankheitsbilder wie Demenz).

Diese Multimorbidität kann den behandelnden Arzt zu einer Polypragmasie (konzeptlose Diagnose und Behandlung) mit kaum vorhersagbaren Wechselwirkungen zwischen den Medikamenten veranlassen. Dabei ist eine Fülle von Faktoren netzwerkartig am Zustandekommen von unerwünschten Wirkungen beteiligt, die altersbedingt zunehmen, wie Organfunktionsund Compliancestörungen. Arzneimittelinduzierte Symptome sind kein seltener Grund für Krankenhauseinweisungen in der Geriatrie. Problematischerweise können diese Symptome als neue eigenständige Erkrankungen fehlinterpretiert werden und zu einer Ausweitung der Medikation führen.

Naturgemäß steigt mit der Zahl der Diagnosen bei älteren Patienten auch die Anzahl der verordneten Medikamente. Nach einer amerikanischen Studie nehmen Patienten (älter als 65 Jahre) in etwa der Hälfte der Fälle 5 und mehr Arzneimittel und in $12 \%$ der Fälle sogar mehr als 10 Arzneimittel ein.

Ein einfacher Erklärungsansatz für diese Polypharmazie besteht in der Leitlinienadhärenz der Ärzte, die ihnen ja als erstrebenswertes Ziel mit normativem Charakter überall angeraten wird: Jede Leitlinie empfiehlt etwa 3 Arzneimittel. Über 80-jährige Patienten haben im Schnitt über 3 Diagnosen. Hieraus ergibt sich eine Arzneimittelzahl von 3 mal 3, also etwa 10 Arzneimittel pro Patient in diesem hohen Lebensalter, was leider der Realität auch entspricht.

Der Hauptdenkfehler in dieser von Leitlinien getriebenen Polypharmazie besteht in der Tatsache, dass es aufgrund der großen Heterogenität der Hochbetagten und der mangelhaften Datenlage keine Leitlinien für dieses Alterskollektiv gibt.

Was hier also zur Adhärenz (Übereinstimmung mit der Therapie) und Polypharmazie führt, nämlich die an Leitlinien orientierte Medizin, weist eindeutige Grenzen in diesem Alterssegment auf. Daher sind auch keine Leitlinienübertretungen nötig, um zu einer rationaleren Therapie im hohen Alter zu kommen.

Als eine Folge dieser Polypharmazie rechnet man für die USA mit immerhin etwa 2,1 Millionen nebenwirkungsbedingten Krankenhauseinweisungen und 100000 Todesfällen pro Jahr auf die damalige Bevölkerung von 265 Millionen Einwohner bezogen. Für Deutschland werden jährlich 20000 Arzneimitteltodesfälle diskutiert. Das Autofahren ist bei 3200 Verkehrstoten pro Jahr in Deutschland inzwischen durch die Errungenschaften der letzten Jahrzehnte (Gurte, Verkehrsführung, strenge Kontrollen) wesentlich sicherer.

Die Nierenfunktion ist bei den meisten betagten Patienten eingeschränkt und damit hier eine regelhaft auftretende Besonderheit für den Arzneimittelstoffwechsel. Z. B. über die Cockcroft-Gault-Formel lässt sich die Nierenfunktion auch ohne aufwändige Parameter (z. B. Sammelurin) abschätzen.

Generell nimmt im Alter das Körperfett auf Kosten des Körperwassers zu, wodurch sich Unterschiede in der Arzneimittelverteilung ergeben. Daraus resultieren gröBere Anteile ungebundener freier Arzneistoffe.

Darüber hinaus unterliegt die Wirkung von Arzneimitteln krankheits- und altersbedingten Veränderungen der End- oder Zielorgane. Sogenannte Arzneimittel-Krankheiten-Interaktionen können hieraus entstehen. Dass über die Nieren ausgeschiedene Arzneimittel bei Nierenschäden nicht oder nur eingeschränkt gegeben werden dürfen, gehört zum Grundwissen jedes Mediziners/Apothekers. Dass aber zahlreiche Arzneimittel selbst auch Nierenschä- den hervorrufen können, die in der Folge auch für akute Vergiftungen verantwortlich sein können, wird viel zu wenig beachtet.

In erster Linie sind hier Schmerz- oder Rheumamittel, sog. nichtsteroidale Antiphlogistika (NSAID), zu nennen, die gerade in Kombination mit anderen, die Nierenfunktion störenden Arzneimitteln wie ACE-Hemmern (Hochdruckmittel) zu akutem Nierenversagen führen können. Bei Hochdruckpatienten macht ein NSAID im Schnitt das Hinzufügen eines weiteren Hochdruckmittels erforderlich, da die Hochdrucktherapie insgesamt an Wirkung verliert.

Ein anderer bekannter, aber häufig unbeachteter Zusammenhang besteht zwischen zahlreichen Arzneimitteln und der Auslösung bzw. der Verschlechterung eines Diabetes mellitus. Zu diesen Arzneimitteln gehören nicht nur reine $\beta$-Blocker und hochdosierte Thiazide, sondern auch Glukokortikoide und andere. Die besondere Empfindlichkeit des geschädigten, aber auch des alten Gehirns gegenüber Beruhigungs- und Schlafmitteln, Morphin und vor allem gegenüber Benzodiazepinen sei hier ebenfalls als bedeutsame Arzneimitteln-Krankheiten-Interaktion erwähnt.

Früh wurde erkannt, dass an Stelle der oft ungenügenden Leitlinienempfehlungen Listenansätze zur Beurteilung der Alterstauglichkeit von Arzneimitteln angewandt werden sollten, die eine schnelle Beurteilung bei ausgedehnten Medikationsschemata ermöglichen. Das erste Hilfsmittel dieser Art war die US Beers-Liste [1], die die alterskritischen Arzneimittel auflistet, deren Anwendung am älteren Patienten nicht erfolgen sollte. Wichtige Substanzgruppen sind hier psychoaktive Pharmaka (Benzodiazepine, Neuroleptika, Trizyklika) oder nichtsteroidale Antiphlogistika, die beim älteren Patienten zu gravierenden Nebenwirkungen wie Verwirrtheit, Stürzen, Kognitionseinschränkung oder gastrointestinalen Blutungen, Bluthochdruck und Herzinfarkten führen können. 13 Jahre später erschien eine deutsche Adaptation dieser Liste, die PRISCUSListe [2]. 
Derartige Negativlisten sind heute in groBer Zahl und mit regionalen Unterschieden verfügbar. Ihnen ist gemeinsam, dass sie primär nur anhand der Arzneimittelliste feststellen, ob unangemessene Arzneimittel (PIM: potentially inappropriate medications) vorhanden sind. In neueren Versionen werden auch Ersatzvorschläge gemacht, die bezüglich der Indikationsprüfung allerdings von der einzigen betrachteten Evidenz ausgehen, nämlich dem Vorliegen eines bestimmten „schlechten“ Arzneimittels, das dann a priori auch eine Indikation haben sollte. Dies ist oft nicht der Fall.

Bislang hat der klinische Einsatz dieser reinen Negativlisten enttäuscht. In einem kürzlich durchgeführten systematischen Review [3] wurden unter 73 Listenansätzen 64 identifiziert, die die Arzneimittelliste in den Vordergrund stellen („Drug-oriented listing approach“ oder DOLA). Nur 9 Listenansätze stellten den Patienten in den Mittelpunkt, um Arzneimittelempfehlungen auf die individuellen Bedürfnisse des Patienten anpassen zu können („patient-in-focus listing approach“ PILA). Die reine Betrachtung der Arzneimittelliste (DOLA), losgelöst von den klinischen Daten des Patienten, ist zwar einladend und attraktiv, da elektronisch leicht zu unterstützen und für epidemiologische Studien leicht zu erfassen; dies führt jedoch nicht zu einer Indikationsüberprüfung. Sie kann daher nicht die Frage beantworten, ob ein „schlechtes“ Arzneimittel, z.B. ein Benzodiazepin zur Behandlung von Schlafstörungen, durch ein besseres ersetzt werden muss, weil es sein kann, dass der Patient überhaupt kein derartiges Arzneimittel benötigt. Dieser grundsätzliche Unterschied zu den meist später entwickelten Positiv/Negativ-Ansätzen (PILA) ist wahrscheinlich auch der wesentliche Grund für die relative klinische Erfolglosigkeit der expliziten Vorgehensweise. In dieser Übersicht waren dann auch klinische Validierungsstudien von PILA signifikant häufiger in Bezug auf eine klinische Besserung der Patienten erfolgreich als solche von DOLA. Dieser Befund entspricht der allgemeinen ärztlichen Erfahrung, dass nur eine vom Patienten ausgehende Beurteilung der Medikation erfolgreich sein kann. Deswegen sind insbesondere zwei Ansätze, die im Jahr 2008 erstmals publiziert wurden,

- Tab. 1 Klassifizierung der Antihypertensiva nach FORTA.

Renin-Angiotensin-System Inhibitoren

ACE-Hemmer $\quad$ A

\begin{tabular}{|l|c|}
\hline Angiotensin-Rezeptor-Antagonisten & A \\
\hline
\end{tabular}

\begin{tabular}{|l|l|}
\hline langwirksame Kalziumantagonisten vom Dihydropyridintyp, z. B. Amlodipin & A \\
\hline
\end{tabular}

Betablocker $\quad$ B

außer Atenolol $\quad$ D

\begin{tabular}{|l|c|}
\hline Diuretika (als Thiazid Indapamid bevorzugt) & B \\
\hline
\end{tabular}

Alphablocker $\quad$ C

Spironolacton $\quad$ C

Moxonidin $\quad$ C

Urapidil $\quad$ C

Aliskiren $\quad$ C

\begin{tabular}{|l|l|} 
Clonidin & D \\
\hline
\end{tabular}

Minoxidil $\quad$ D

Calciumantagonisten vom Verapamiltyp

$\mathrm{D}$

hervorzuheben, die als PILA sowohl Negativ- als auch Positivempfehlungen kombiniert berücksichtigten. Das START/ STOPP-Projekt [4] listet Kriterien für „schlechte“ Arzneimittel, deren Anwendung beendet werden soll (STOPP), aber auch positive Handlungsanweisungen (START), die zu einer besseren Nutzung von Chancen für einen älteren Patienten führen. Das FORTA-Prinzip [5] ist die bislang einzige Positiv/Negativ-Bewertung einzelner Arzneimittel oder Arzneimittelgruppen in Bezug auf altersrelevante Diagnosen und kategorisiert diese Arzneimittel von A (sehr vorteilhaft) über B (vorteilhaft) und $C$ (kritisch, nur in Ausnahmefällen zu verabreichen) bis $D$ (praktisch immer zu vermeiden). Dieses Prinzip wurde zur Entwicklung einer Arzneimittelliste (FORTAListe) verwendet und hat in insgesamt drei Delphiprozessen zu einer jetzt 296 Bewertungen in 30 Indikationen umfassenden Liste geführt [6]. Beide Systeme sind inzwischen in klinischen Interventionsstudien erfolgreich evaluiert worden. Zuerst konnte in der VALFORTA-Studie [7] gezeigt werden, dass eine FORTA-Implementierung auf geriatrischen Stationen zu einer hochsignifikanten Verbesserung der Medikationsqualität nach dem FORTAScore (Summe aus Über- und Untertherapiefehlern, nur durch eine Positiv/NegativListe zu leisten) führt. Diese Studie war die erste, die eindeutig auch zu klinisch positiven Endpunkteffekten geführt hat: Bei nur 16-tägiger Intervention an im Schnitt 81- jährigen Patienten sank die Rate von Arzneimittelnebenwirkungen gegenüber einer nach geriatrischen Standardbedingungen therapierten Kontrollgruppe hochsignifikant $a b$, und dies bei einer NNT (Number Needed to Treat) von nur fünf! Des Weiteren verbesserte sich der Barthel-Index signifikant stärker in der FORTA-Gruppe als in der Kontrollgruppe. Danach erschien eine ganz ähnliche Studie mit vergleichbaren Endpunkteffekten zum START/STOPP-System [8]; dies unterstreicht die große Bedeutung des positiven Anteils in diesen kombinierten Positiv/Negativ-Bewertungssystemen.

Die Besonderheiten in der Arzneimitteltherapie des alten Patienten sollten insgesamt jedoch nicht dazu verleiten, dieser hochrelevanten Zielgruppe hoffnungsvolle medikamentöse Therapieansätze vorzuenthalten. Wichtig ist es, sich auf essenzielle Therapien zu konzentrieren und somit die Zahl der Medikamente, wenn möglich, zu vermindern. Insbesondere die Behandlungen des Bluthochdrucks und der Cholesterinerhöhung sind inzwischen auch für ältere Patienten als äußerst erfolgreich belegt. Hier ist als Beispiel eine Liste der in der Hochdrucktherapie üblichen Medikamente nach der FORTA-Klassifikation wiedergegeben, die dem Arzt eine schnelle Orientierung ( 8 min Kontaktzeit in der primärärztlichen Versorgung!) erlaubt ( $\triangleright$ Tab. 1): 
Die gesamte FORTA-Liste ist frei zugänglich unter http://www.umm.uni-heidelberg.de/ag/forta/. Außerdem existiert jetzt für alle Handy-Systeme eine kostenlose APP (FORTA in den entsprechenden AppStores eingeben).

Leider fehlen in vielen Bereichen noch Studiendaten zur Arzneimitteltherapie älterer Patienten, da sie aus Zulassungsstudien für Arzneimittel häufig ausgeschlossen werden. Angesichts der großen zahlenmäßigen Bedeutung der älteren Patienten und der anspruchsvollen Therapiebedingungen ist dies ein gravierender Mangel, der energischer angegangen werden sollte.

Abschließend soll nochmals betont werden, dass jede Arzneimittelanwendung ein Individualexperiment darstellt, das trotz aller Informationen nur unter genauer klinischer Verlaufskontrolle sicher durchzuführen ist!

Wer "scharfe“" Messer - und das sind viele der hochwirksamen modernen Medikamente - führen will, muss mit ihnen umzugehen lernen und sich der möglichen Gefahren bewusst sein.

\section{Interessenkonflikt}

Der Referent war von 2004 bis 2006 aufgrund einer Tätigkeit für die Fa. AstraZeneca beurlaubt. Seit 01.01.2007 ist er wieder Professor für Klinische Pharmakologie an der Universität Heidelberg in Mannheim. Vor und nach dieser Zeit war und ist er als Gutachter, Berater und Referent für SanofiAventis, Bayer, Boehringer Ingelheim, Novartis, Takeda, Roche, Pfizer, Bristol-Myers Squibb, Daichii-Sankyo, Lilly, Otsuka, NovoNordisk, Shire and LEO Pharma tätig.

\section{Autorinnen/Autoren}

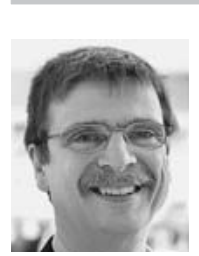

\section{Martin Wehling}

Prof. Dr. med., Direktor des Instituts für Klinische Pharmakologie der Medizinischen Fakultät Mannheim, Universität Heidelberg

\section{Korrespondenzadresse}

Prof. Dr. med. Martin Wehling

Direktor des Instituts für Klinische Pharmakologie

Medizinische Fakultät Mannheim

Universität Heidelberg

Theodor-Kutzer-Ufer 1-3

68167 Mannheim

KPM@medma.uni-heidelberg.de
Literatur

[1] Beers MH. Explicit criteria for determining potentially inappropriate medication use by the elderly. An Update. Arch Intern Med 1997; 157: 1531-1536

[2] Holt S, Schmiedl S, Thürmann PA. Potentially inappropriate medications in the elderly: The Priscus List. Dtsch Ärztebl Int 2010; 107: 543-551

[3] Pazan F, Kather J, Wehling M. A systematic review and novel classification of listing tools to improve medication in older people. Eur J Clin Pharmacol 2019; 75: 619-625

[4] O'Mahony D, O'Sullivan D, Byrne S et al. STOPP/START criteria for potentially inappropriate prescribing in older people: version 2. Age Ageing 2015; 44: 213-218

[5] Wehling M. Arzneimitteltherapie im Alter: Zu viel und zu wenig, was tun? Ein neues Bewertungssystem: fit for the aged FORTA. Dtsch Med Wochenschr 2008; 133: 22892291

[6] Pazan F, Weiss C, Wehling M. The FORTA (Fit fOR The Aged) List 2018: Third Version of a Validated Clinical Tool for Improved Drug Treatment in Older People. Drugs Aging 2019; 36(5):481-484

[7] Wehling M, Burkhardt $\mathrm{H}$, Kuhn-Thiel A et al. VALFORTA: a randomized trial to validate the FORTA (Fit fOR The Aged) classification. Age Ageing 2016; 45: 262-267

[8] O'Connor MN, O'Sullivan D, Gallagher PF et al. Prevention of hospital-acquired adverse drug reactions in older people using screening tool of older persons' prescriptions and screening tool to alert to right treatment criteria: a cluster randomized controlled trial. J Am Geriatr Soc 2016; 64:1558-1566 